\title{
Cirurgia combinada de catarata e glaucoma com ponto escleral perilímbico: técnica cirúrgica e resultados a longo prazo
}

\author{
Combined cataract and glaucoma surgery with scleral \\ peri-limbic suture: surgical technique and long-term results
}

Luciano Sólia Násser', Pedro Eleutério dos Santos Neto², Lívia Máris Ribeiro Paranaíba ${ }^{3}$, Luciana dos Mares Guia Ribeiro ${ }^{4}$, Luciano Pimenta de Figueiredo5 ${ }^{5}$ Hercílio Martelli Júnior ${ }^{3}$

\section{Resumo}

Objetivo: Analisar os resultados da cirurgia combinada de catarata e glaucoma (FACO-TREC) e apresentar a técnica com o ponto escleral perilímbico. Métodos: Estudo retrospectivo por levantamento de prontuários com informações sobre a pressão intraocular, acuidade visual, medicação hipotensora antes e depois da cirurgia e descrição do ato cirúrgico combinado (FACO-TREC), com acompanhamento mínimo de seis meses. Foram selecionados 10 pacientes com glaucoma e catarata (15 olhos), acompanhados de janeiro de 2005 a junho de 2007, no Departamento de Glaucoma da Santa Casa Olhos de Montes Claros (MG). A cirurgia de TREC utilizada foi a de base fórnix, sem uso de Mitomicina C, com a incisão principal da cirurgia de catarata realizada no mesmo sítio da TREC. A confecção do ponto escleral perilímbico se dá como uma sutura que se inicia na esclera nua, passa por cima do retalho escleral e é ancorada na esclera do outro lado do retalho. A conjuntiva é suturada com pontos simples por sobre o ponto. Resultados: A acuidade visual, após seis meses da cirurgia mostrou-se melhor em 86,6\% (13) dos olhos, inalterada em 6,66\% (1 olho) e pior em 6,66\%. A pressão intraocular média pré-operatória foi de 18,02 $\mathrm{mmHg}$, a pós-operatória após 6 meses foi de 15,06 $\mathrm{mmHg}$. Cinquenta por cento dos olhos operados ficaram livres dos colírios. Conclusão: Conclui-se que o ponto escleral perilímbico apresentou resultados favoráveis na obtenção de uma melhora na acuidade visual, redução da pressão intraocular e uma diminuição significativa no uso de drogas hipotensoras à custa de um número muito pequeno de complicações.

Descritores: Glaucoma; Extração de catarata; Facoemulsificação; Trabeculectomia; Técnicas de sutura

\section{ABSTRACT}

Purpose: To analyze the results of combined cataract and glaucoma surgery and to present the technique with scleral peri-limbic suture. Methods: Retrospective study with review of the data about intraocular pressure, visual acuity, antihypertensive medication before and after surgery and description of the combined surgery technique, with follow up of six months. We selected 10 patients with glaucoma and cataract (15 eyes) followed up from January 2005 to June 2007 from Santa Casa Olhos de Montes Claros $(M G)$. The glaucoma surgery was fornix-based, without use of mitomycin $C$, with the main cataract surgery incision performed on the same site The preparation of the scleral peri-limbic suture is given as a suture that begins on bare sclera, passes over the scleral flap and is anchored in the sclera on the other side of flap. The conjunctiva is sutured over the peri-limbic point. Results: The visual acuity after six months of surgery was better in $86.6 \%$ of eyes, unchanged in $6.66 \%$ (one eye) and worse in 6.66 . The mean preoperative IOP was $18.02 \mathrm{mmHg}$, after six postoperative months was $15.06 \mathrm{mmHg}$. Fifty percente of the eyes were free of eye drops. Conclusion: We conclude that scleral limbic suture showed favorable results in obtaining an improvement in visual acuity, reduction of intraocular pressure and a significant decrease in the use of hypotensive drugs at the expense of a very small number of complications.

Keywords: Glaucoma; Cataract extraction; Phacoemulsification; Trabeculectomy; Suture techniques

\footnotetext{
'Programa de Pós-Graduação (Mestrado) em Ciências da Saúde, Universidade de Montes Claros (Unimontes) - Montes Claros (MG), Brasil.

${ }^{2}$ Mestre em Ciências da Saúde pela Universidade de Montes Claros (Unimontes) - Montes Claros (MG), Brasil;

${ }^{3}$ Programa de Pós-Graduação em Ciências da Saúde, Universidade de Montes Claros - (Unimontes) - Montes Claros (MG), Brasil;

${ }^{4}$ Universidade de Montes Claros - (Unimontes) - Montes Claros (MG), Brasil;

${ }^{5}$ Santa Casa Olhos de Montes Claros - Montes Claros (MG), Brasil.

Trabalho realizado no Departamento de Glaucoma da Santa Casa Olhos de Montes Claros - Montes Claros (MG), Brasil
}

Os autores declaram inexistir conflitos de interesse

Recebido para publicação em 29/7/2011 - Aceito para publicação em 2/3/2012 


\section{INTRODUÇÃO}

C atarata e glaucoma são, respectivamente, a primeira e terceira causa de cegueira no mundo ${ }^{(1)}$. Devido ao aumento da sobrevida da população e a escassez de recursos suficientes para o seu tratamento, o glaucoma tem sido responsável por um aumento significativo dos casos de cegueira $^{(2)}$ e sua associação com catarata no paciente idoso tem sido cada vez mais frequente ${ }^{(3)}$.

A cirurgia de trabeculectomia (TREC) vem sofrendo, nos últimos tempos, importantes modificações nos seus tempos cirúrgicos. Tais modificações visam aumentar o sucesso funcional da cirurgia ao atuarem, basicamente, em duas vertentes: manter um fluxo ideal pela fístula e diminuir as complicações pós-operatórias, principalmente as imediatas ${ }^{(4)}$. Recentes estudos trouxeram novos avanços na TREC para evitar as complicações referentes à bolha: uso de antiproliferativos aplicados mais posteriormente, aumento do tamanho do flap escleral e a utilização de suturas ajustáveis. Tais modificações visam direcionar o fluxo para a região posterior do fórnix conjuntival ${ }^{(4)}$.

Considerando-se os grandes avanços técnicos nos últimos anos, tem-se cada vez mais optado pelo tratamento cirúrgico combinado da catarata e glaucoma. Entre as vantagens de uma cirurgia única destaca-se a redução do risco cirúrgico relacionado à anestesia e à infecção, bem como os custos relacionados ao procedimento e cuidados pós-operatórios ${ }^{(5)}$.

No período pós-operatório inicial, é importante que a pressão intraocular PIO não caia demasiadamente no olho operado, causando hipotonia. Para diminuir o risco de filtração em excesso pode-se lançar mão de suturas de contenção que mantenham uma ferida apertada e uma Po pós-operatória mais alta, que ao serem desfeitas, fazem com que a PIO chegue ao limite pré-estabelecido, uma vez que a cicatrização da conjuntiva e cápsula de Tenon já consigam restringir o fluxo do humor aquoso(6).

O objetivo deste trabalho é analisar os resultados da cirurgia combinada de catarata e glaucoma (FACO-TREC) e apresentar uma técnica cirúrgica alternativa no arsenal terapêutico atual: o ponto escleral perilímbico.

\section{Métodos}

Trata-se de um estudo retrospectivo através de levantamento de prontuários que continham a história pregressa e atual do glaucoma, bem como todas as informações referentes à PIO, acuidade visual (AV), medicação hipotensora utilizada antes e depois da cirurgia e descrição detalhada do ato cirúrgico combinado (FACO-TREC), com acompanhamento mínimo de seis meses a partir da cirurgia. Foram excluídos pacientes com cirurgias oculares prévias, antecedentes de trauma ocular e prontuários que continham um exame ocular incompleto no seguimento pós-operatório. Assim, foram selecionados 10 pacientes com diagnóstico confirmado de glaucoma e catarata, totalizando 15 olhos, acompanhados de janeiro de 2005 a junho de 2007, no Departamento de Glaucoma da Santa Casa Olhos de Montes Claros, em Minas Gerais (MG). Todos os pacientes haviam assinado o termo de consentimento livre e esclarecido, bem como o termo de autorização para divulgação científica das informações e imagens médicas advindas do tratamento. Os

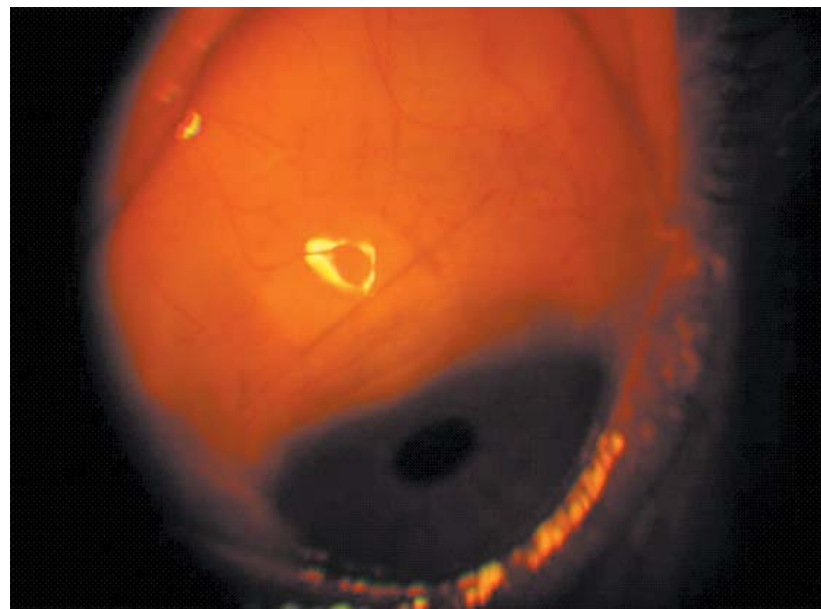

Figura 1: Ponto longitudinal peri-límbico subconjuntival

pacientes incluídos no estudo apresentaram uma acuidade visual, no olho afetado, com correção $\leq 20 / 50$, catarata nuclear 1 a $3+$, com opacidade de eixo e glaucoma avançado com PIO alvo ameaçada. A PIO pré-operatória foi calculada pela média das três últimas consultas antes da cirurgia, tomadas no período da manhã, entre 8 e $11 \mathrm{~h}$. Para efeito de análise estatística, a AV foi transformada em escala decimal. O número de colírios foi comparado antes e após a cirurgia.

Técnica cirúrgica: a anestesia foi realizada por injeção peribulbar. A cirurgia de TREC utilizada foi a de base fórnix, sem uso de Mitomicina C, com a incisão principal da cirurgia de catarata realizada no mesmo sítio da TREC e feita por um mesmo cirurgião. Em todos os casos foi utilizada lente intraocular dobrável colocada dentro do saco capsular e não houve ruptura da cápsula posterior. A confecção do ponto longitudinal perilímbico se dá como uma sutura que é ancorada na esclera nua, passa por cima do flap escleral e é ancorada na esclera do outro lado do flap escleral. A tensão do ponto longitudinal se soma com as suturas isoladas na porção posterior do flap escleral. A conjuntiva é suturada com pontos simples por sobre o ponto longitudinal (Figura 1).

O pós-operatório foi padronizado da seguinte maneira: $1^{\circ}$ dia: avaliação da PIO, bolha, câmara anterior, LIO; $3^{\circ}$ dia: medida da PIO; $5^{\circ}$ dia: medida da PIO e remoção ou não do ponto escleral; $10^{\circ}$ ao $15^{\circ}$ dia: medida da PIO e remoção ou não dos pontos posteriores do flap escleral; $1^{\circ}$ mês: medida da PIO; $3^{\circ}$ mês: medida da PIO e refração; $6^{\circ}$ mês: medida da PIO, exame do fundo de olho e após um ano, exame do fundo de olho com nova refração e medida da PIO.

Como critérios de sucesso ou insucesso, os parâmetros utilizados estão descritos a seguir. Sucesso: Po maior que $8 \mathrm{mmHg}$ e menor ou igual $15 \mathrm{mmHg}$, sem uso de colírios após 6 meses da cirurgia. Sucesso relativo: PIO alvo atingida à custa de colírios antiglaucomatosos em número menor ao utilizado antes da cirurgia. Insucesso: PIO alvo alcançada à custa de colírios antiglaucomatosos em número maior ou igual ao utilizado antes da cirurgia.

Drogas antiglaucomatosas foram reinstituídas caso a PIO apresentasse risco para o nervo óptico, ou seja, nos casos com dano avançado e Po maior do que $15 \mathrm{mmHg}$. Dados referentes a acuidade visual, PIO e uso de colírios 
Gráfico 1

Medidas da acuidade visual (AV) antes e após a cirurgia

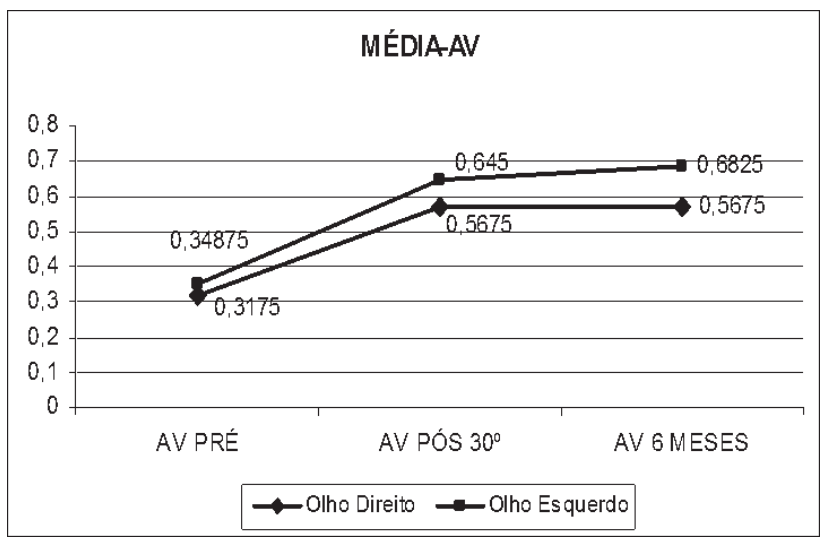

hipotensores foram organizados em um banco e comparados no pré, no pós-operatória precoce e tardio, para estudo. A análise estatística foi realizada através do programa SPSS, versão 17 (SPSS, Inc, Chicago, IL). Para comparar dados, foi utilizado o teste T de Student, considerando um nível de significância de $5 \%$ e um intervalo de confiança de $95 \%$.

\section{$\underline{\text { ResultadOS }}$}

A idade dos pacientes variou de 59 a 73 anos (média de 71,53 anos). Foram estudados 15 olhos de 10 pacientes, destes 66,6\% (10 olhos) eram da raça branca e 33,3\% (5 olhos) da raça negra. Quanto à classificação do glaucoma, $77,7 \%$ (12) dos olhos apresentaram glaucoma primário de ângulo aberto (GPAA) e 22,3\% (3 olhos) apresentaram glaucoma primário de ângulo fechado (GPAF).

A acuidade visual, após seis meses da cirurgia mostrou-se melhor em 13 olhos $(86,6 \%)$, inalterada em um olho $(6,66 \%)$ e pior em um olho $(6,66 \%)$. A acuidade visual préoperatória variou de $0,1(20 / 200)$ a $0,4(20 / 50)$ com média de 0,33 e a pós-operatória (após 6 meses) variou de 0,1 (20/200) a $1,0(20 / 20)$ com média de 0,625 , sendo uma diferença significativa $(\mathrm{p}=0,04)$ (Gráfico 1$)$. A acuidade visual final foi maior ou igual a $20 / 40 \mathrm{em} 86,6 \%$ dos olhos.

A PIO pré-operatória variou de 12 a $24 \mathrm{mmHg}$ (média de $18,02 \mathrm{mmHg}$ ), a pós-operatória no $30^{\circ}$ dia variou de 10 a $18 \mathrm{mmHg}$ (média de $14,25 \mathrm{mmHg}$ ) e a pós-operatória final (após 6 meses) variou de 13 a $18 \mathrm{mmHg}$ (média de 15,06 $\mathrm{mmHg}$ ), uma variação significativa $(\mathrm{p}=0,041)$ (Gráfico 2). Após 6 meses da cirurgia, 33,3\% dos olhos apresentaram uma PIO menor ou igual a $15 \mathrm{mmHg}$ sem colírios e $20 \%$ uma PIO menor ou igual a $15 \mathrm{mmHg}$ com colírios, porém, em número menor que no pré-operatório.

O número de colírios antiglaucomatosos no pré-operatório variou de 2 a 3 tipos diferentes de colírios e no pósoperatório final (após 6 meses da cirurgia) variou de 0 a 2 colírios (Gráfico 3). Cinco olhos (33,3\%) obtiveram sucesso dentro dos critérios acima estabelecidos $(\mathrm{p}=0,047)$. Sucesso relativo foi observado em 3 olhos $(20 \%)$ e insucesso em 2 olhos $(13,33 \%)$.
Gráfico 2

\section{Valores da pressão intra-ocular (Po) antes e depois da cirurgia}

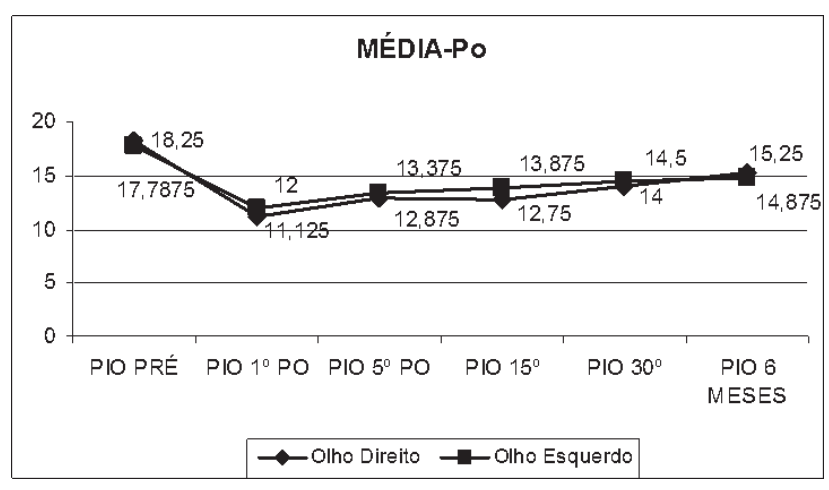

\section{Gráfico 3}

Uso de colírios antes e após a cirurgia

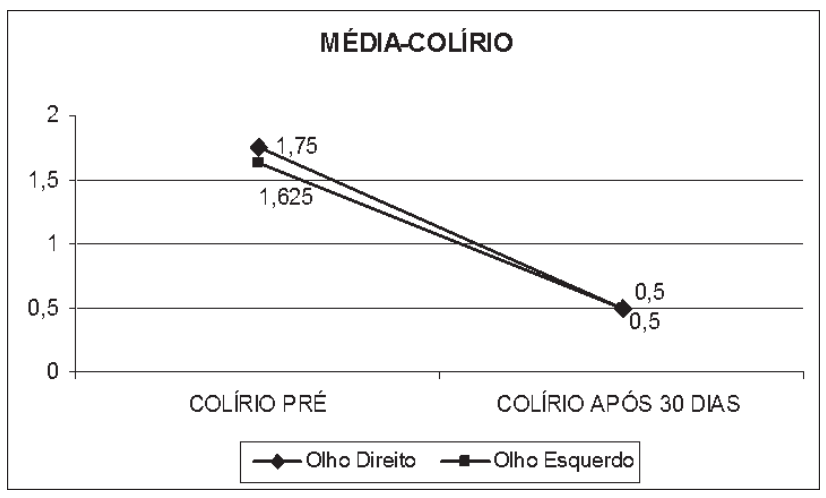

Observou-se hifema em um olho $(6,66 \%)$ e opacidade de cápsula posterior em um olho $(6,66 \%)$. O hifema recebeu conduta expectante e evoluiu para resolução espontânea. A opacidade de cápsula posterior foi tratada com capsulotomia por Nd-YAG laser no segundo mês pós-operatório, sem intercorrêncas.

Realizou-se a lise do ponto escleral perilímbico no $5^{\circ}$ dia de pós-operatório de 12 olhos (80\%). A massagem do globo ocular foi realizada no primeiro mês em 8 olhos $(53,3 \%)$, nos casos em que a pressão estava acima do esperado e ou não se observava fluxo de humor aquoso subconjuntival mesmo após a remoção do ponto longitudinal. Nestes casos em que a massagem resultou em diminuição da PIO ( 8 olhos ou 53,3\%) foi realizada a lise do ponto posterior do flap escleral com laser de argônio entre o $15^{\circ} \mathrm{e}$ $20^{\circ}$ dia de pós-operatório.

\section{Discussão}

Neste estudo, os resultados obtidos com a FACOTREC sem uso de antimetabólito mostraram um bom controle da PIO com baixo índice de complicações, embora estudos consistentes evidenciassem benefício no uso de mitomicina $\mathrm{C}^{(7)}$. As complicações cirúrgicas em FACO-TREC 
mais comumente descritas na literatura são: hifema, opacidade da cápsula posterior, hiperfiltração, presença de seidel, hipotonia, maculopatia hipotônica, descolamento seroso da coróide, endoftalmite e baixa acuidade visual ${ }^{(8)}$. As cirurgias combinadas têm possibilitado um bom controle da PIO no pós-operatório imediato e a longo prazo, baixa necessidade de uso de colírios hipotensores, satisfatória recuperação da acuidade visual e a vantagem de tratar duas patologias em um mesmo ato cirúrgico ${ }^{(5)}$.

A acuidade visual teve considerável melhora na maioria dos pacientes (Gráfico 1). Nota-se uma melhora da AV que se mantém constante após 6 meses.

Observou-se uma diminuição estatisticamente significante $(\mathrm{p}=0,047)$ no número de colírios antes e após a cirurgia (Gráfico 2). Cinco olhos (33,3\%), correspondente a metade dos pacientes, ficaram livres de colírios. Em um estudo prospectivo de metodologia semelhante foi mostrado que $89 \%$ dos pacientes operados de FACO-TREC ficaram independentes quanto ao uso de colírios hipotensores ${ }^{(8)}$. Outro importante estudo evidenciou um índice de $67 \%{ }^{(9)}$. Concluise que é importante aprimorar esta técnica para que se aproxime dos resultados publicados em outros trabalhos.

Somando-se o número de pacientes com sucesso e sucesso relativo, isto é, pacientes que atingiram PIO inferior a $\mathrm{PIO}$ alvo sem colírios somados àqueles que a atingiram com uso de colírios em número menor do que antes da cirurgia, atingem-se $86,7 \%$ de sucesso. Outros estudos mostram índices de sucesso semelhantes, embora existam muitas variações metodológicas e amostras díspares entre cada um ${ }^{(3,10)}$. No presente estudo, 86,7\% dos olhos apresentaram, após seis meses de acompanhamento, uma PIO inferior a $15 \mathrm{mmHg}$, valor satisfatório para esta amostra com dano glaucomatoso bastante avançado.

Com relação às complicações pós-operatórias surgidas em $13,33 \%$ dos olhos, trata-se de um índice bem inferior quando comparado com outros trabalhos ${ }^{(11-13)}$. A realização das cirurgias por um profissional experiente em FACO-TREC, a não utilização de antimetabólitos e a presença do ponto longitudinal perilímbico podem estar associados a estes bons resultados.

Diferentemente da sutura de compressão e suas variações $^{(14)}$, o ponto escleral perilímbico é realizado sobre o retalho escleral e abaixo da conjuntiva, próximo ao limbo. Portanto ele funciona como uma barreira inicial ao fluxo do humor aquoso sem causar compressão entre a conjuntiva e a esclera. Uma vez que ele é removido, aumenta-se o fluxo de humor aquoso para o espaço subconjuntival, que ficou preservado desde a sua confecção.

\section{Conclusão}

Este estudo mostrou que a cirurgia combinada de FACO-TREC por meio de uma mesma incisão, sem uso de antimetabólitos em pacientes com glaucoma e catarata resulta em melhora da acuidade visual, redução da pressão intraocular a longo prazo e uma diminuição significativa no uso de drogas hipotensoras. Como os resultados mostraram um número reduzido de complicações intra e pós-operatórias, pode-se inferir que o uso do ponto escleral perilímbico apresentou resultados favoráveis no controle da PIO e ma- nutenção de uma boa acuidade visual, já no período pósoperatório imediato de FACO-TREC.

Sugere-se que mais estudos utilizando um maior número de pacientes devem ser realizados para confirmar a eficiência desta técnica em comparação às técnicas comumente utilizadas.

\section{REFERÊNCIAS}

1. Resnikoff S, Pascolini D, Etya'ale D, Kocur I, Pararajasegaram R, Pokharel GP, Mariotti SP. Global data on visual impairment in the year 2002. Bull World Health Organ. 2004;82(11):844-51.

2. Quigley HA. Number of people with glaucoma worldwide. Br J Ophthalmol. 1996;80(5):389-93. Comment in Br J Ophthalmol. 1996;80(5):385-6. Br J Ophthalmol. 1997;81(1):93.

3. Gutemberg GCV, Silva Filho FJ, Rehder JRCL. Complicações pós-operatórias precoces de trabeculectomia com mitomicina, em pacientes portadores de glaucoma primário de ângulo aberto. Rev Bras Oftalmol. 2010;69(2):100-3.

4. Schuman JS. Surgical management of coexisting cataract and glaucoma. Ophthalmic Surg Lasers. 1996;27(1): 45-59.

5. Jones E, Clarke J, Khaw PT. Recent advances in trabeculectomy technique. Curr Opin Ophthalmol. 2005;16(2):107-13.

6. Friedman DS, Jampel HD, Lubomski LH, Kempen JH, Quigley $\mathrm{H}$, Congdon N, et al. Surgical strategies for coexisting glaucoma and cataract: an evidence-based update. Ophthalmology. 2002;109(10):1902-13. Comment in Ophthalmology. 2004;111(2):408-9.

7. Susanna Júnior R. Mudando conceitos na cirurgia de trabeculectomia. Ocular Surg News (Latin America Edition). 2009:12-15. Disponível em:http://www.osnsupersite.com/ view.aspx? rid=37995

8. Jampel HD, Friedman DS, Lubomski LH, Kempen JH, Quigley $\mathrm{H}$, Congdon $\mathrm{N}$, et al. Effect of technique on intraocular pressure after combined cataract and glaucoma surgery: An evidence-based review. Ophthalmology. 2002;109(12):2215-24; quiz 2225, 2231.

9. Costa VP, Smith M, Spaeth GL, Gandham S, Markovitz B. Loss of visual acuity after trabeculectomy. Ophthalmology. 1993;100(5):599-612.

10. Palmberg P. Combined cataract and glaucoma surgery with mitomycin. Ophthalmol Clin North Am. 1995;8:365-81.

11. Wand M. Combined phacoemulsification, intraocular lens implant, and trabeculectomy with intraoperative mitomycinC: comparison between $3.2-$ and $6.0-\mathrm{mm}$ incisions. J Glaucoma. 1996;5(5):301-7.

12. Caporossi A, Casprini F, Tosi GM, Balestrazzi A. Long-term results of combined 1-way phacoemulsification, intraocular lens implantation, and trabeculectomy. J Cataract Refract Surg. 1999;25(12):1641-5.

13. Prata JA Jr, Seah SK, Minckler DS, Baerveldt G, Lee PP, Heuer DK. Postoperative complications and short-term outcome after 5-Fluorouracil or mitomycin-C trabeculectomy. $\mathrm{J}$ Glaucoma. 1995;4(1):25-31.

14. Zacchei AC, Palmberg PF, Mendosa A, Robinson JC. Compression sutures: a new treatment for leaking or painful blebs. Invest Ophthalmol Vis Sci.1996;37(3):S444.

\section{Autor correspondente:}

Luciano Sólia Násser

Rua Monte Pascoal, 57- Ibituruna

CEP 39401-000 - Montes Claros (MG), Brasil

Email: solianasser@gmail.com 